\title{
Advanced nonlinear signal processing in silicon-based waveguides
}

\author{
P. Petropoulos, M. Ettabib, K. Bottrill, C. Lacava, \\ F. Parmigiani, K. Hammani* \\ Optoelectronics Research Centre, University of \\ Southampton, Southampton SO17 1BJ, United Kingdom \\ Email: pp@orc.soton.ac.uk
}

\author{
M. Brun, P. Labeye, S. Nicoletti
}

CEA Leti MINATEC Campus, F-38054 Grenoble 9, France

\author{
A. Bogris ${ }^{* *}$, A. Kapsalis, D. Syvridis \\ Department of Informatics and Telecommunications, \\ National and Kapodistrian University of \\ Athens,Panepistimiopolis, Ilissia, 15784, Athens, Greece
}

"Now at Laboratoire Interdisciplinaire Carnot de Bourgogne (ICB), UMR 6303 CNRS-Université de Bourgogne, 9 av. A. Savary, BP 47 870, F-21078 Dijon Cedex, France ${ }^{* *}$ Also, with Department of Informatics, Technological Educational Institute of Athens, Aghiou Spiridonos, 12210 Egaleo, Athens, Greece
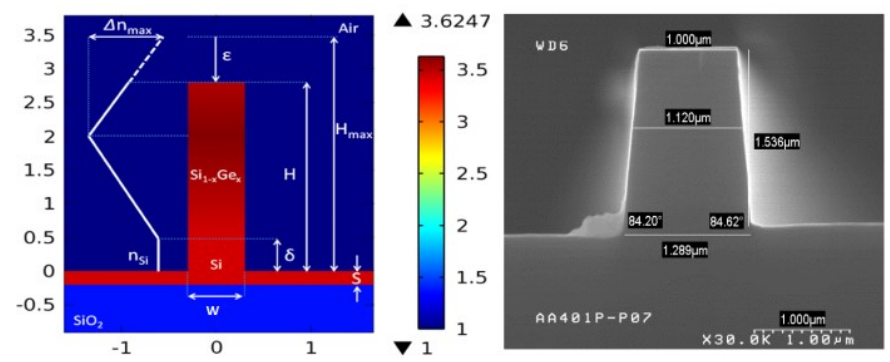

Fig. 1. (Left) Design of a silicon germanium waveguide exhibitting a graded refractive index variation; (right) scanning electron microscopy image of the fabricated waveguide [6].

\section{REFERENCES} is the nonlinear elements in processing systems. Smalldimension waveguides based on silica have recently shown great potential in this direction, and a number of important demonstrations have been reported using pure silicon waveguides (see e.g [1]). Here we review recent progress, and focus on the potential of silicon germanium waveguides, in which the inclusion of germanium allows flexibility in the manipulation of the optical properties of the nonlinear element $[2,3]$ - see Fig.1. Recent applications in wavelength conversion [4], signal regeneration [5] as well as supercontinuum generation [6] are reviewed.

\begin{abstract}
processing based on compact waveguides fabricated mainly using silicon germanium alloys. Applications include supercontinuum
\end{abstract}

Keywords—silicon photonics; nonlinear optics; optical signal vastly from advances in optical glasses and developments in optical fibre design and fabrication, it is widely accepted that there is still a lot to be desired from materials that are to
This work was supported by the European Communities Seventh Framework Program FP7/2007-2013 under Grant 288304 (STREP CLARITY), and the U.K. Engineering and Physical Sciences Research Council under the Programme Grants "Transforming the Future Internet: The Photonics Hyperhighway" and "Silicon Photonics for Future Systems".
[1] M. A. Foster, A. C. Turner, J. E. Sharping, B. S. Schmidt, M. Lipson, and A. L. Gaeta, "Broad-band optical parametric gain on a silicon photonic chip," Nature, vol. 441, pp. 960-963, 2006.

[2] K. Hon, R. Soref, and B. Jalali, "The third-order nonlinear optical coefficients of $\mathrm{Si}, \mathrm{Ge}$, and $\mathrm{Si}_{1-\mathrm{x}} \mathrm{Ge}_{\mathrm{x}}$ in the midwave and longwave infrared," J. Appl. Phys., vol. 110, p. 011301, 2011.

[3] K. Hammani, M. A. Ettabib, A. Bogris, A. Kapsalis, D. Syvridis, M. Brun, et al., "Optical properties of silicon germanium waveguides at telecommunication wavelengths," Optics Express, vol. 21, pp. 16690 16701, Jul 152013.

[4] M. A. Ettabib, K. Hammani, F. Parmigiani, L. Jones, A. Kapsalis, A. Bogris, et al., "FWM-based wavelength conversion of 40 Gbaud PSK signals in a silicon germanium waveguide," Optics Express, vol. 21, pp. 16683-16689, Jul 152013.

[5] M. Ettabib, F. Parmigiani, A. Kapsalis, A. Bogris, M. Brun, P. Labeye, et al., "Record phase sensitive extinction ratio in a silicon germanium waveguide," in CLEO'2015, San Jose, CA, 2015, p. paper STh1.

[6] M. A. Ettabib, L. Xu, A. Bogris, A. Kapsalis, M. Belal, E. Lorent, et al., "Telecom to Mid-Infrared Supercontinuum Generation in a Silicon Germanium Waveguide," in $O F C^{\prime 2015}$, Los Angeles, CA, 2015, p. paper Tu2C.5. 